O

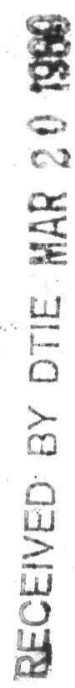

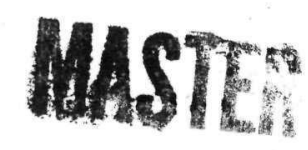

OPERATIONS PIAN

FOR

OFE-SITE SURVEILLANCE FOR THE LAUNCH OF A SNAP-19

May 10,1968

Prepared by

Southwestern Radiological Health Laboratory

U. S. Public Health Service

Department of realth, Education, and Welfare

Las Vegas, Nevada 


\section{DISCLAIMER}

This report was prepared as an account of work sponsored by an agency of the United States Government. Neither the United States Government nor any agency Thereof, nor any of their employees, makes any warranty, express or implied, or assumes any legal liability or responsibility for the accuracy, completeness, or usefulness of any information, apparatus, product, or process disclosed, or represents that its use would not infringe privately owned rights. Reference herein to any specific commercial product, process, or service by trade name, trademark, manufacturer, or otherwise does not necessarily constitute or imply its endorsement, recommendation, or favoring by the United States Government or any agency thereof. The views and opinions of authors expressed herein do not necessarily state or reflect those of the United States Government or any agency thereof. 


\section{DISCLAIMER}

Portions of this document may be illegible in electronic image products. Images are produced from the best available original document. 
TABLE OF CONTENTS

$\begin{array}{cl}\text { I. } & \text { INTRODUCTION } \\ \text { II. } & \text { RESPONSIBILITIES } \\ \text { III. } & \text { OPERATING PROCEDURES }\end{array}$

A. Interagency Relations I

B. Personnel

C. Communications

D. Field Plan

E. Reports

Appendix A Selected Sample Control and Analytical procedures Used at the Southwestern Radiological Health Laboratory

$A-1$

Appendix A -1 Laboratory procedures $\quad$ A - I

Appendix A -2 Rapid Method for the Determination of Gross

Alpha in Sea Water and Natural Water Samples

A -2

Appendix A.-3 A Rapid Method for the "Estimation" of Deposited Alpha Activity on Soil, Vegetation and Similar Sample Types

A -4

Appendix A-4 A Rapid Method for the Estimation of Deposited Alpha Activity on Flora

A -6

Appendix A -5 Radioassay Procedures

A -7

Appendix B

General Field Procedures

$\mathrm{B}-1$

Appendix B-1 Monitoring and Record Keeping

$\mathrm{B}-1$

Appendix B-2 Field Unit Check List

$\mathrm{B}-3$

Appendix B-3 Instrument Instructions

B -4

Appendix B-4 Sample Identification and Marking

$\mathrm{B}-8$ 


\author{
OPERATIONS PIAN \\ FOR \\ OFF-SITE SURVEILTANCE \\ FOR \\ TIIE LAUNCH OF A SNAP-19
}

\title{
T. INTRODUCTION
}

The Southwestern Radiological Health Laboratory (SWRHL) \} of the National Center for Radiological Health, U.S. Public Health Service will provide off-site monitoring for the launch of the SNAP-19 from Vandenberg AFB, California.

\section{RESPONSIBILITTES}

The SWRHL will provide the services listed below, with respect to the off-site area. 1

1. Maintain a record of background environmental radioactivity levels during the launch activities to assure protection of the general population and environment.

2. Provide liaison regarding health protection between local and state officials and officials of the lst Strategic Air Division.

3. Provide community information to assure the public that their safety will be protected during the launch.

4. Identify specific hazards, if any, associated with the lauich and assist in carrying out appropriate protective measures.

\section{OPERATING PROCEDURES}

\section{A. Interagency Relations}

The interagency relations program shall be the direct responsibility of the Public Health Service Project officer. He will contact local, state, and military leaders throughout the off-site area to describe the surveillance activities. He or his representative will follow up all initial contacts to answer questions which may ariseand provide continuity of information.

I That area outside the Vandenberg AFB. The area of immediate interest includes Santa Barbara County. 
B. Personne1

Persomnel will be assigned from the Southwestern Radiological Health Laboratory. This staff will receive special alpha monitoring training at the sWrHL as well as receive actual alpha monitoring experience at the Nevada Test Site (NTS). The staff will be in the field at D-3 days for orientation, communications check and bucksround determination. The Director of SWRHL will be in charge of 211 PHS personnel and will be currently advised by the project Officer. Assignments of personnel will be made prior to the start of the activities.

C. Communications

Two-way radio communication is planned through radibs installed in vehicles. Standby points will be previously selected in order to maintain continuous communications. Radio communications are through a simplex system, but terrain precludes direct contact and an airborne voice relay is planned. Preliminary investigation will establish the locations for optimum communication.

Each standby unit will inform the field office of the number of a phone near the standby position. In the event of radio failure, commercial telephones will be utilized.

D. Field plan

On launch day four mobile units will be on standby at strategic points throughout Santa Barbara County, two additional units will be on standby in the Vandenberg area for commitment as events dictate. It is anticipated that the units will be located at Santa Maria, Vandenberg Village, Gaviota, and Buellton.

Each mobile unit will be equipped with suitable monitoring instrumentation for the measurement of alpha radiation as well as the de- 
tection of the low energy gamma radiation from specific types of alpha omitting nuclides; sample containers for the collection of water, vegetation and soil; portable air samplers for unattended air sample operation in remote areas; the necessary supplies and forms for proper documentation of pHS field activities. In case of accident cach unit will establish an air samplex at a previously designated location and initiate action as directed by the project officer. Six air samplers will be operated continuously for 24 hours through the day of the launch. Sampling sites will be at Vandenberg Air Force Base, Lompoc, Surf, Jalama, Buellton, and Gaviota. These sites will be operated without regard to incidents.

In case of accident, the Public Health Service personnel will document any radioactivity which is released. They will also be available to provide assistance and consultation if an unforeseen emergency occurs. In addition, they will collect local envirommental samples for laboratory documentation of the extent of contamination.

In case of accident, monitors will determine the extent of contamination using survey meters. More accurate assessment of the contamination will be obtained through laboratory analysis of samples collected from the field. This laboratory analysis will consist of the gross alpha procedures identified as Apprndix A.

E. Reports

A consolidated summary of SWRHL activities giving actions taken, areas covered, and field results will be prepared at the conclusion of the launch operation. This report will be submitted to appropriate government agencies and state and local health departments. 


\section{APPENDIX $\Lambda$}

Selected Sample Control and Analytical procedures used at the Southwestern Radiological Health Laboratory.

APPENDIX $\triangle-1$

\section{Laboratory Procedures}

Sample control. One person will be responsible for receiving and logging samples as they are brought in from the ficld. Samples will be assigned a number by the person in charge of logging. The off-Site data sheet will be used for air samples. Simple logging of location and time are all that is initially required. 


\title{
APPHNDIX $\Lambda-2$
}

\begin{abstract}
A Rapid Method for the Determination of Gross Alpha
in Sea Water and Natural Water Samples
\end{abstract}

Introduction

This method describes a rapid procedure for the determination of soluble and suspended alpha activity in sea water. This method is also applicable to other natural water samples. The recovery, using a plutonium nitrate spike, averaged $80 \%$.

Sample

Results by this method are entirely dependent upon the sample. A representative sample must be secured, and the analysis made as soon as possible.

Apparatus and Reagents

Beaker, graduated, 1,000 $\mathrm{ml}$

Counter, laboratory, any suitable alpha counter

planchet, stainless steel, 2 inch with $5 / 16 \mathrm{rim}$

Tube, immersion, 60 M (Corning Catalog \#39535 or equivalent)

pH Paper

Ammonium Hydroxide, Concentrated

Cerric Nitrate, $0.5 \mathrm{M}$

Fexric Chloride, $1 \mathrm{M}$

Nitric Acid, concentrated, $3 \mathrm{~N}$

Procedure

1. A 1,000 $\mathrm{ml}$ sample of water is measured in a graduated beaker.

2. Add, with stirring, $20 \mathrm{ml}$ concentrated $\mathrm{HNO}_{3}, 0.5 \mathrm{ml}$ of $0.5 \mathrm{M}$ Cerric nitrate, and 2 drops of 1 M ferric chloride - in that order. NOTE: A blank determination should be run on the Cerric nitrate. 
3. Adjust to a pII of 9 , with pH paper, using concentrated NI OIl, stir and add $2-3$ drops excess.

1. Let precipitate form. Filter, using an immersion tube, discard the supernate。

5. Dissolve the precipitate by carefully washing the walls of the beaker and face of the immersion tube with a minimum of $3 \mathrm{~N}$ HNO.

6. Transfer quantitatively to a tared planchet using $3 \mathrm{~N} \mathrm{HNO}_{3}$, evaporate, flame and weigh. Submit for alpha counting. 
APPENDIX A-3

A Rapid Method for the "Estimation" of Deposited

Alpha Activity on Soil, Vegetation and

Similax Sample Types

Introduction

This method describes a procedure for the "estimation" of alpha activity in soil and vegetation samples. Leafy floxa samples will be done by Method 3 , Appendix $B-4$.

The sample is leached in a beaker with nitric acid and the sample is filtered. Evaporation of a suitable portion of the filtrate is followed by counting. A portion of the solids is tested for any remaining alpha activity。 When the method was run as specified, a yield recovery of $80 \%$ average was obtained for soluble plutonium nitrate spikes.

Apparatus and Reagents

Beaker, 1,000 ml

Counter, laboratory, any suitable low level alpha counter

Filtex paper, \#2

Filter flask, 1,000 m

Buchner funne1, 3"

Flask volumetric, 1,000 ml

Hot plate, electric

Planchet, stainless steel, $2^{\prime \prime}$ diameter

Stirring rods, mechanical or manual

Nitric acid, $3 \mathrm{~N}$

\section{Procedure}

1. Transfer the entire weighed sample (200 g or less) to a 1 liter. beaker. Add $400 \mathrm{ml} 3 \mathrm{~N} \mathrm{HNO}_{3}$ and place on hot plate.

2. Bring and keep solution near boiling for 30 minutes with stirring. 
3. Cool and filter through a Buchner funnel with \#2 filter paper under vacuum. Save filtrate.

4. Repeat leach a second time combining filtrates. Dilute filtrates to $1,000 \mathrm{ml}$.

5. Take a suitable aliquot (Note \#I) of filtrate to give a residue upon evaporating in planchet of not more than $100 \mathrm{mg}$.

6. Evaporate aliquot to dryness, flame, weigh, and submit for alpha counting。

NOTES:

1. The "suitable aliquot" in step \#5 can be determined by running evaluation samples from the area of sampling.

2. The leached soil or vegetation should be dried and several $80-100 \mathrm{mg}$ portions counted on a planchet in a proportional counter to estimate any large amount of insoluble alpha activity。

3. The occurrence of natural a activity in soil, and unreproducible sampling conditions introduce errors of unacceptable magnitude when soil reporting results are in units of activity per unit area. 
APPENDIX A-1

A Rapid Method for the Estimation of Deposited Alpha Activity on Flora

Introduction

This method describes a washing procedure to remove deposited activity on flora. A sample of 50-100 $\mathrm{g}$ is placed in a $400 \mathrm{ml}$ beakex and washed with I $\mathrm{N} \mathrm{HNO}_{3}$ in an ultrasonic cleaner. Evaporation of the wash water depending upon solids present, is followed by counting. A $90 \%$ average recovery is obtained for this procedure.

Apparatus and Reagents

Ultrasonic cleaner - tank unit, Harrison Model 751 or equivalent.

Beaker, $400 \mathrm{~m} 1$

Counter, laboratory

Nitric Acid, $1 \mathrm{~N}$

Planchet, stainless steel, 2" diameter

Procedure

1. Weigh a suitable sample (50-100 g) and transfer to a $400 \mathrm{ml}$ beaker.

2. Add $200 \mathrm{ml} 1 \mathrm{~N} \mathrm{NHO}_{3}$ and place in a suitable ultrasonic cleaner tank. Operate unit for 10 minutes.

3. Carefully remove flora, washing with $1 \mathrm{~N} \mathrm{HNO}_{3}$. Evaporate wash solution to near dryness and transfer to a planchet, evaporate to dryness, flame and weigh. Submit for alpha counting.

NOTES :

If the wash solution contains a large amount of solids, an aliquot should be taken for counting. Maximum residue on the planchet should not exceed $100 \mathrm{mg}$. 
APPENDIX A-5

Radioassay procedures

Gross alpha determination will be made using a Beckman Wide-Beta proportional counter. Calculations to be used with the counter are as follows:

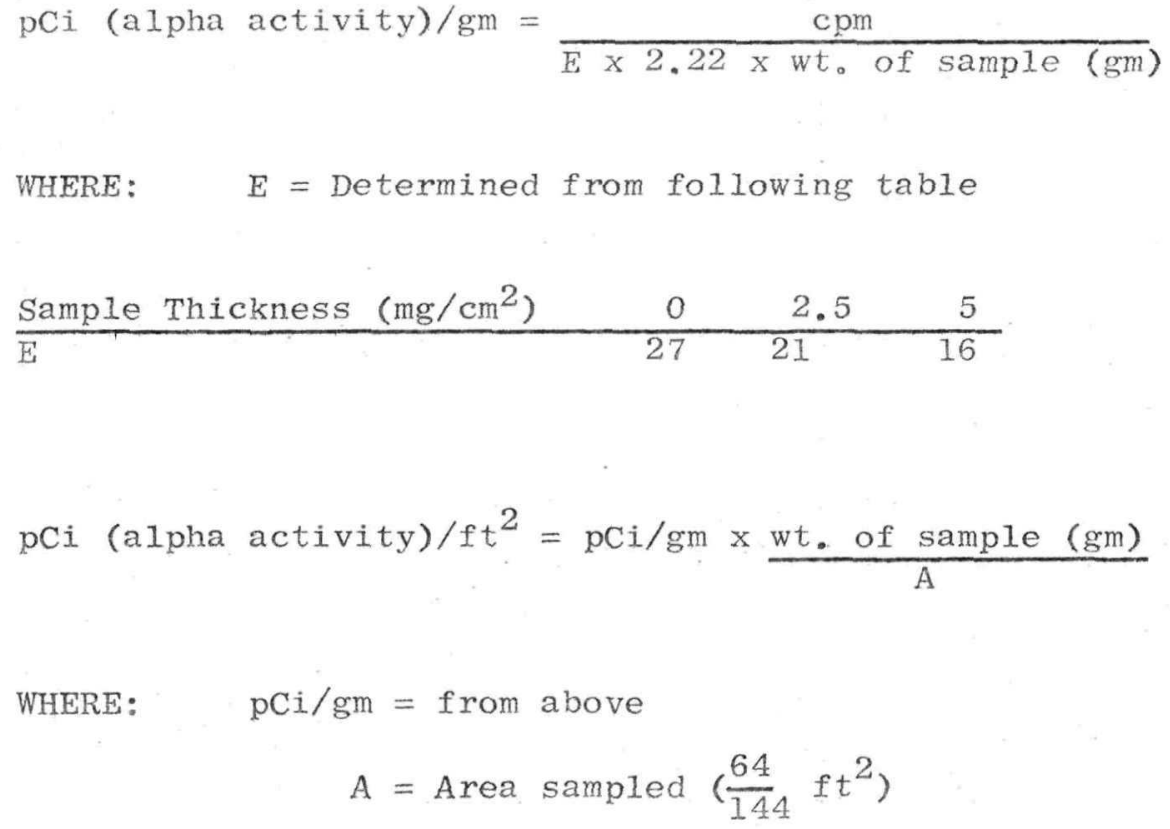


APPENDTX B

General. Field procedures

APPENDIX B -1

Monitoring and Record Keeping

Field units will compile a comprehensive record of all their activities. The record will consist of the date, the location, the times of action, the times of receipt of radio or telephone messages, the point of contact in terms of telephone number and location. They will also maintain a record of general readings obtained with the Low energy gamma probe (PG-1) on the PAC 1-S instrument for background purposes prior to and during the launch. These values will be recorded by place, time and level with instrument number identification. This field log will contain specific data on samples taken and also summarize conversations held between PHS personnel and local individuals.

The PAC 1-S is a sensitive alpha survey instrument capable of giving reliable readings when used with caution. Since this is a scintillation counter, it is susceptible to light leaks. Before being taken to the field, all instruments will have been checked for light leakage. To avoid puncturing the light seal by stones, sticks or sharp edges of surface material, survey personnel will handle the instruments with extreme caution. Since it is an alpha measuring instrument, the head must be placed in close proximity to the source of material because of the limited range of alpha particles in air. It should be carefully laid on a flat surface, that has no protrusions which may puncture the light seal, and allowed to come to equilibrium. For those activities which are below the sensitivity of the integrating circuitry, a count can be obtained by use of a stop watch and the headset. Audible pulses can be then totaled for a known interval and the number of counts per minute determined.

This technique of monitoring does not lend itself to rapidly closing upon a contamination source. Therefore, for initial detection, a low energy gamma detector is provided which can be used to approach the source. However, 
the sensitivity of the gamma probe is much less than that of the flat area alpha probe and for the delineation of action areas the flat area probe will be used.

All instruments should be periodically checked against the source provided with the monitoring kit. 


\section{APPEND IX B -2}

Field Unit Check List

Eberline Emergency Monitoring Kit (PAC 1-S with Alpha scintillator probe and low-energy gamma probe)

2 Portable air samplers w/propane tanks

Glass fiber and membrane filters

Clippers and knife for vegetation sampling

Plastic bags for packaging soil and vegetation samples

Gallon bottles and cardboard boxes for water samples

Data sheets for air samplers

Monitoring $\log$ sheets

Glassine envelopes for packaging air sampler filters

Felt tip marking pens and grease pencils

Shovel and miscellaneous small tools

Field log book 


\section{APPENDIX B -3}

Instrument Instructions

The PAC 1-S instrument, as dolivered, has the alpha probe connected and calibrated. The tape on the side of the alpha probe lists the meter reading which should be obtained when the alpha check source is centered in the protective cover and the probe is resting on the source. The count rate recorded on the probe may differ from that listed on the source. In that event, use the value on the probe.

There are two sources provided with each kit.

1. Radium source, approximately 5 microcuries, for use with E-500B.

2. Uranium source for alpha probe and low energy gamma probe $(P G-1)$.

The backside has a count rate and serial number. The plateside or front side appears as uncovered metal, be careful not to touch with anything.

To check for light leaks, place on $X-1$ scale, turn the face to the sun (or other strong light source) and tip in various positions watching indication. Cable noise will cause minor needle movement while rotating the probe. The needle will remain high while the probe is held steady if a Iight leak is present. If a majox meter indication is observed, hold the probe against the body to cut out all light. If the reading drops, the needle deflection was crused by a light leak. When a light leak is observed in direct light, turn the probe away from the light source to determine if leaks are minor and if the instrument is usable. A minor leak would be reilected under 500 counts per minute. With a major leak, hold the probe facing direct light and cover with cardboard or other shield. Gradually open up the area to light by sliding the shield across the probe to locate the grid area involved. Recover and slide off at $90^{\circ}$ to original direction to isolate the portion of the grid area involved.

$$
\mathrm{B}-4
$$


paint this area with black lacquer provided with the kit. In using the lacquer, wipe the major portion off the brush and apply a thin coat to a small area.

After repaix, repeat the pertinent instructions to determine the effectiveness of the procedure. 
Changing to $\mathrm{PG}-1$ (Low energy gamma probe)

1. Turn instrument off.

2. Remove alpha probe at probe end of cable.

3. Commect PG-1 probe to cable.

4. Instrument may peg on $X-1$ scale so turn to $X-10$ scale.

5. Remove case with Ra source to a point at least 15 fect away.

6. Remove cap from discriminator.

7. Using small Eberline key chain screwdriver found in each kit, turn discriminator in clockwise direction to the stop, then back off (counter clockwise) $30^{\circ}$ ( $2 / 3$ of one division)。

8. Check response by placing alpha check source on flat surface with metal up, cover with top of plastic petri dish (the top has the larger diameter), rest the end of $\mathrm{PG}-1$ probe on top of petri dish cover. Instrument should read value written on tape stuck to side of PG-1 probe. This is an operational check and not a calibration.

9. Replace discriminator cap。

Use of $\mathrm{PG}-1$ Probe

This probe is not and cannot be calibrated to give quantitative information on contamination. It is only an indicator or a search tool. Suspected contamination can be approached holding the cable and allowing the probe to hang about one foot from the ground. Indications of activity should be reassessed with the alpha probe.

Failure of the PG-1 to detect activity is not absolute proof that contamination is not present. However, it does provide information that action levels have not been exceeded. Further evaluation of possible contamination must be conducted by use of the alpha probe.

Replacing Alpha Probe

1. Turn instrument off.

2. Remove PG-1 from probe end of cable. 
3. Connect alpha probe to cable.

1. Turn instrument on to $\mathrm{x}-10$ scale.

5. Remove discriminator cap.

6. Place alpha source with metal up inside circle in protective cover.

7. Place probe ovex source in protective cover.

8. With small screwdriver, slowly turn discriminator counter clockwise until meter movement is first observed. Continue turning counter clockwise in small increments allowing meter movement to stablize after each increment until meter indicates the counts per minute rate for source as marked on side of alpha probe. Ise the value placed on the probe rather than. the count rate given on the source. There may be a difference between the two values. Check to see that the source sexial number matches the serial number recorded with the count rate on the side of the probe.

9. Replace the discriminator cap.

Use of Alpha Probe

1. The range of ${ }^{238} \mathrm{Pu}$ alpha is less than $4 \mathrm{~cm}$ in air, therefore, the alpha probe must be close to the surface being evaluated.

2. The instrument is of the scintillation type and the light seal (window area) which will admit alpha particles and exclude light is easily torn or punctured.

3. Only qualitative information can be obtained from non-uniform surfaces.

4. Quantitative information must be obtained from flat surfaces which extend beyond the probe on all sides. The probe must rest on or be adjacent to the flat surface for quantitative reading.

5. For most accurate quantitation the meter should remain horizontal.

6. After placing the probe, allow the needle to stabilize. The time necessary for stabilization is greater on the lower scales.

7. Recorded values should be the average of 5 readings taken one at each corner and one in the center of a square meter area.

8. Each of the 5 readings used to develop the above average value should be the mean between upper and lower meter fluctuations observed for each probe position. 


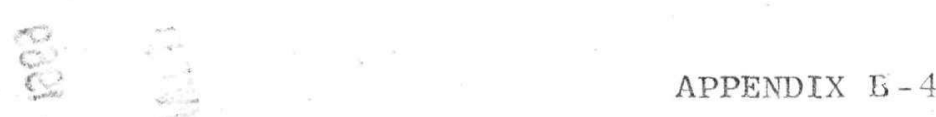

$\lim _{\rightarrow \infty}$

Sample Identification and Marking

marked for subsequent identification. The sample information necessary is:

1. The description of the location sufficiently detailed for a second person to return to that area and take a duplicate sample.

2. The time and date of collection.

3. The type of sample.

4. The person collecting the sample.

5. Any additional remarks regarding condition of the soil, the sample, or the weather which may be pertinent to analysis or interpretation.

This information should be recorded in the monitoring $\log$ and also on a sample identification sheet which will accompany the sample。 